Kong. Res. J. 1(2): 10-15, 2014

Kongunadu Arts and Science College, Coimbatore

\title{
CONSTRUCTION OF MINIMUM CONNECTED DOMINATING SET IN A GRAPH AND ITS APPLICATION IN WIRELESS SENSOR NETWORKS
}

\author{
Vijayalakshmi, D*. and R. Kalaivani \\ Department of Mathematics, Kongunadu Arts and Science College, Coimbatore - 641029. \\ E.mail: vijikasc@gmail.com
}

\section{ABSTRACT}

Finding Minimum Connected Dominating Set in a graph is one of the important problems in graph theory. It is largely applied in Wireless Sensor Networks (WSN). It acts as a virtual backbone in WSNs. algorithms of finding such minimum connected dominating set was studied. A new algorithm for constructing a routing in WSN using one of the algorithms is proposed and implemented using java language. The sample outputs are also included.

Keywords: Connected Dominating Set, wireless sensor networks, virtual backbone.

\section{INTRODUCTION}

A backbone in a Wireless Sensor Network (WSN) reduces the communication overhead, increases the band width efficiency, decreases the overall energy consumption and thus increases network operational life. The nodes in a wireless sensor network forward data towards a sink via other nodes. The limited resources on the nodes require minimum energy to be spent in this energy consuming task. This necessitates a virtual backbone that can minimize the number of hops required to reach the sink assuming that all nodes have equal transmission range. In the wireless domain, this backbone is a minimum connected dominating set (MCDS). Away from an element of the subset forms a dominating set $S$. A connected dominating set (CDS) $C$ of $G$ is a dominating set $S$ in hitch all the elements are connected i.e. it induces a connected graph. The nodes in $C$ are called dominators and the other nodes which are one hop away from $C$ are dominates. To minimize the number of hops, the minimum CDS is chosen as the backbone. The backbone is the smallest CDS and every node is adjacent to this virtual backbone. Once data is received by a dominator, it is relayed through the MCDS towards the sink for minimum hop communication. Since the nodes have equal transmission range, the CDS has to be determined for Unit Disk Graph.

\section{AUXILIARY DEFINITION}

In order to develop the algorithm, we state some definition and introduce some terminology relevant to the paper.

2.1. Dominating Set - Dominating Set for a graph $\mathrm{G}=$ $(V, E)$ is a subset $D$ of the Vertex Set V such that each vertex uÎV is either in $\mathrm{D}$ or adjacent to some vertex $\mathrm{V}$ in D. The elements of dominating set are called dominators. Examples of dominating set in a graph $\mathrm{G}$ are given below:

Figure $1 .\{1,3\},\{2,3,5\}$ and $\{1,2,3,4\}$ are Dominating sets

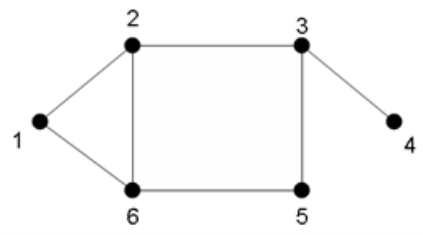

Figure 2. $\{4,6\},\{1,5,7\}$ and $\{4,5,6\}$ are Dominating Sets

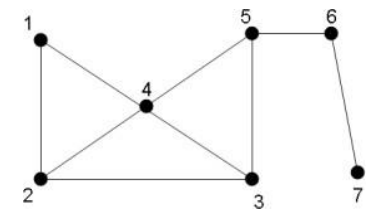

2.2. Connected Dominating Set - A Connected Dominating Set (CDS) of a graph $G=(V, E)$ is a set of vertices with two properties:

1. D is a dominating set in $\mathrm{G}$.

2. D induces a connected subgraph of G.

In Fig. $1,\{2,3,5\}$ and $\{1,2,3,4\}$ are

Connected Dominating Sets. Similarly in Fig. 2, \{4, 5, $6\}$ is a Connected Dominating Set.

2.3. Minimum Connected Dominating Set - A minimum Connected Dominating Set (MCDS) is a connected dominating set with smallest possible cardinality among all the CDSs of G. As in Figs. 1 and $2,\{2,3,5\}$ and $\{4,5,6\}$ are Minimum Connected Dominating Sets respectively.

2.4. Convex hull - the convex hull for a set of points $\mathrm{X}$ in real vector space is the minimum convex set 
containing $\mathrm{X}$. it is also called convex envelop and denoted by $\mathrm{CH}(\mathrm{X})$. It is represented by a sequence of the vertices of the line segment forming the boundary of the convex polygon.

\section{A POWER AWARE MINIMUM CONNECTED DOMINATING SET FOR WIRELESS SENSOR NETWORKS.}

A backbone in a wireless sensor networks (WSN) reduces the communication overhead, increases the bandwidth efficiency, decreases the overall energy consumption and thus increases network operational life. The nodes in a wireless sensor networks forward data towards data a sink via other nodes.

The limited resources on the nodes require minimum energy to be spent in this energy consuming task. This necessitates a virtual backbone that can minimize the number of hopes required to reach the sink assuming that all nodes have equal transmission range. In the wireless domain, this backbone is a minimum connected dominating set (MCDS).

\subsection{Finding a Routing in WSN Using Power Aware Minimum Connected Dominating Set}

Here, we are presenting an algorithm for a finding a routing in WSN using power aware minimum connected dominating set is presented. The proposed algorithm is divided into two phases. In the phase a random network is generated and in the second phase routing is achieved using the algorithm.

\subsection{Generation of Random Network}

Random graphs were introduced by erdos and renyi in the late fifties. The random graph model generates a graph that has a number has a number of nodes which are connected randomly by undirected edges. A random graph is obtained by starting with a set of $\mathrm{n}$ vertices and adding edges between them at random. Here a random nodes are initially placed in the specified area $\mathrm{m} \times \mathrm{m}$ and at each iteration edges are created randomly using those nodes.

\subsection{MCDS Construction}

Dominating set is constructed which consists of minimum number of nodes. This phase consists of following steps:

1. An arbitrary number say id is assigned to each Node in the graph $G(V, E)$

2. Each node is assigned white color
3. The node $u$ with maximum degree is taken from $\mathrm{G}(V, E)$ and colored as black, i.e. Dominator

4. All the neighbor nodes of the node $u$ are Colored i.e. Dominatee

5. Do step 3-4 till all the nodes in the graph $G(V, E)$ are colored either as black or gray.

PHASE-I $(G(V, E))$

$D \leftarrow \pitchfork$

if ( $\forall x \in V \mathrm{x}$.color is black or gray)

then exit

else $\mathrm{u} \leftarrow$ MAX-DEGREE $(\mathrm{V})$

u.color $\leftarrow$ black

$D \leftarrow D \cup\{\mathrm{u}\}$ for $\forall x \in N\{u\}$

do x.color $\leftarrow$ gray

\section{Phase-1 Algorithm}

PHASE - $1((G(V, E))$

Explanation of Algorithm Phase-I: Each node in the graph is assigned an arbitrary number as id. Each node is assigned with white color in the beginning. A node $x \in G(V, E)$ s.t. $x$ has maximum degree is determined; if two nodes have same degree i.e. maximum then choose a node having minimum $i d$. Let that node be $u$. color node $u$ as black i.e. Dominator and this node is added into list of Dominating Set i.e. D. All the neighbors of node u i.e. Dominates are colored as gray so that they are not considered in dominating set. The same is repeated for remaining uncolored graph until all the nodes get colored. The above given algorithm can be understood with the help graph shown in figure 4. In this graph initially all nodes are considered as white nodes. Select node which has maximum degree. Node 8 and node 12 both have maximum degree i.e. five. according to this algorithm in case of tie of the degree, lowest id is considered first, so node 8 is selected and colored black and all its neighboring nodes i.e 6,7,9,10and 11 are colored gray.

Fig 3.1. Initial Graph

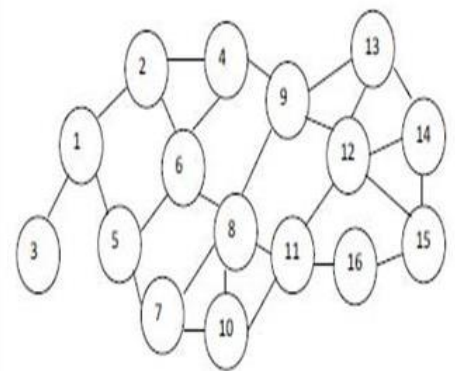


Fig.3.2 .Node 8 selected as domnting node

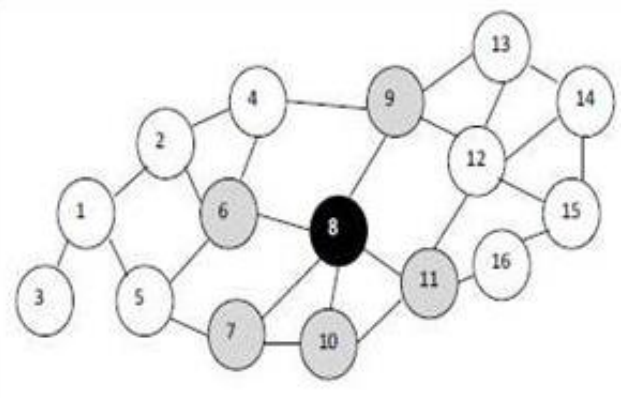

Similarly in next step node 12 is considered which has maximum degree and colored black and its neighbours i.e. 9,11,13,14 and 15 colored as gray.

Fig 3.3. Node 8 and 12 selected as dominating set

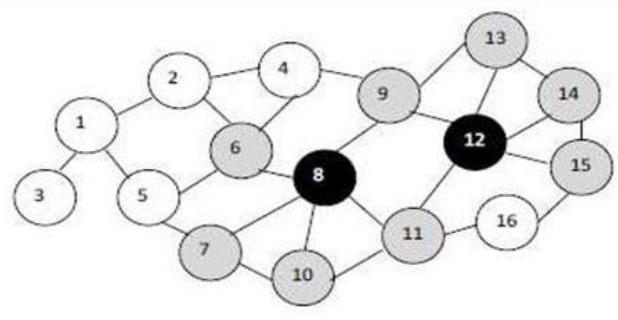

In next step same procedure repeat and node 1 is selected which has maximum degree with lowest i.d., colored black and all its neighbors 2,3 and 5 colored as gray.

Fig 3.4: Nodes 1.8.12 selected as dominating set

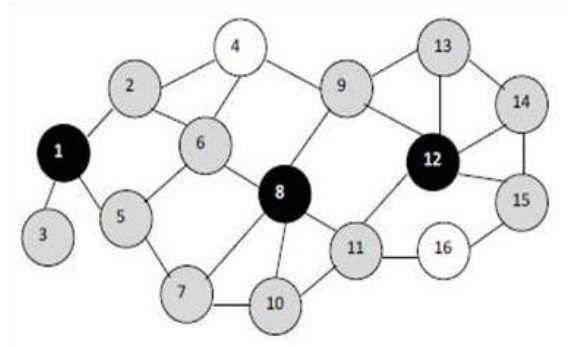

Fig 3.5. Nodes $1,4,8,12$ and 16 are dominatig set nodes

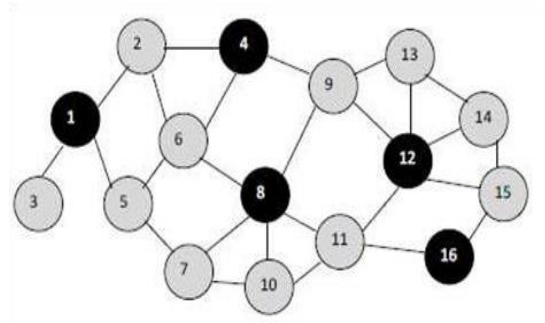

\section{Phase II. Determination of Connectors}

In this phase, set of connectors $B$ is found such that all the nodes in dominating set $D$ gets connected. Let a black node be a node in $D$ and a dark gray node represent a node in $B$. a node in $B$ is connected by at most $K$ nodes in the graph $G(V, E)$. Set of dark gray nodes with given $D$ could be found using Steiner tree. It is a tree, interconnecting all the nodes in $D$ by adding new nodes between them. The nodes that are in the Steiner tree but not in set $D$ are called Steiner nodes. In the MCDS set, the number of Steiner nodes should be minimum. After this steps CDS is constructed, which will consist of black and dark gray nodes. Let the constructed CDS be set $F$. This involves the following steps.

1. Select a gray node which is connected to Maximum $(K)$ number of black nodes, set

Its color as dark gray

2. Check whether the Dominating Set $D$

3. if $D$ gets connected stop

4. else go to step 1 with $K-1$ number of black nodes

Phase - II Algorithm

$B \leftarrow \phi$

for $i \leftarrow$ Kto2

do

while there exist a node $v$ which is connected to $K$ adjacent black nodes

do

$B \leftarrow B \cup\{v\}$

if $D$ is connected

then exit

after completing the dominating set nodes $1,4,8,12$ and 16 select a node which connected maximum number of black nodes i.e. node 11 and colored s dark gray

\section{Fig 3.6. Connecting node 11}

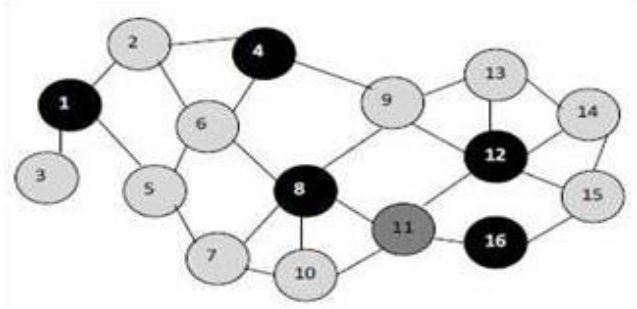


Repeat this step until all black nodes are connected. All the black nodes and all the dark gray nodes are form the connecting dominating set.

Fig 3.7. Connecting nodes 11 and 9

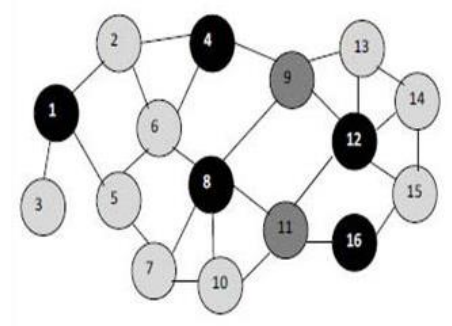

Fig 3.8. Connecting nodes 9,11 and 2

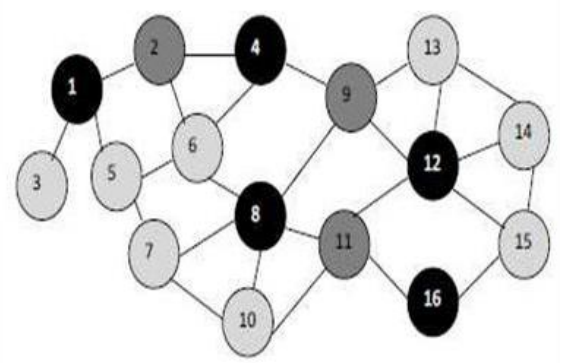

So final CDS which covers nodes $1,2,4,8,9,11,12$ and 16 . This can be shown in fig 3.9.

Fig 3.9: cds nodes are 1,2,4,8,9,11,12 and 16.

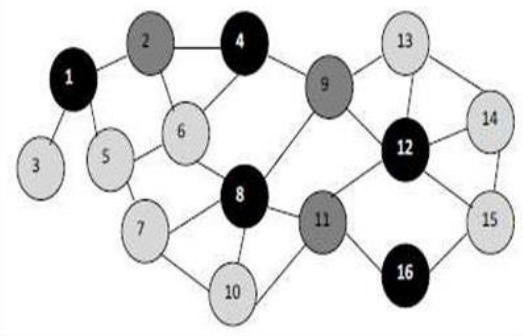

\section{Phase III: Pruning}

This is the pruning phase. In this phase, redundant nodes are deleted from the CDS constructed in phase II, to obtain the MCDS. The following steps are

1. Select a minimum degree node $u$ from $F$

2. check if $N[u]$ is subset of $N[1]$ and $N[2]$ and $\ldots N[n]$ where $i$ belongs to $F-\{u\}$

3. if step 2 returns true then remove node $u$ and Goto step 1

4. Otherwise do not remove node $u$ and Goto step 1
Fig 3.10. $\{1,2,4,9,8,11,12\}$ is finial MCDS backbone

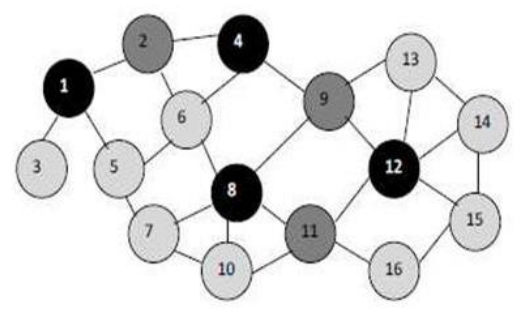

For this CDS graph select a node with the minimum degree among black nodes and dark gray nodes and delete it. Node 16 is deleted because node 16 is subset of $N$ (12) N (11). The degree of node 16 is less than degree of the other CDS nodes. So final CDS is found after pruning process covers nodes are $1,2,4,8,9,11$ and 12 . This final CDS is minimum and known as MCDS

\subsection{Algorithm proposed}

In this project we are proposing an algorithm to find a routing in WSN. This algorithm consists of two phases. In phase I, a random network with $\mathrm{n}$ nodes which are randomly placed in specified area $\mathrm{m} \mathrm{x} \mathrm{m}$ is generated. In phase II by applying the algorithm proposed minimum connected dominating set if found, using which routing is achieved for the network

Phase I

A random network with $\mathrm{n}$ nodes which are randomly placed in specified area $\mathrm{m} \mathrm{X} \mathrm{m}$ is generated

\section{Phase II}

By applying the algorithm proposed in [6] Minimum Connected Dominating Set is found, using which routing is achieved for the network.

\section{Implementation}

Random graphs were introduced by Erdos and Renyi in the late fifties. The random graph model generates a graph that has a number of nodes which are connected randomly by undirected edges. The algorithm is implemented and tested using JAVA. The sample outputs of the proposed algorithm are given here. 
Fig 3.11. Position of the nodes.

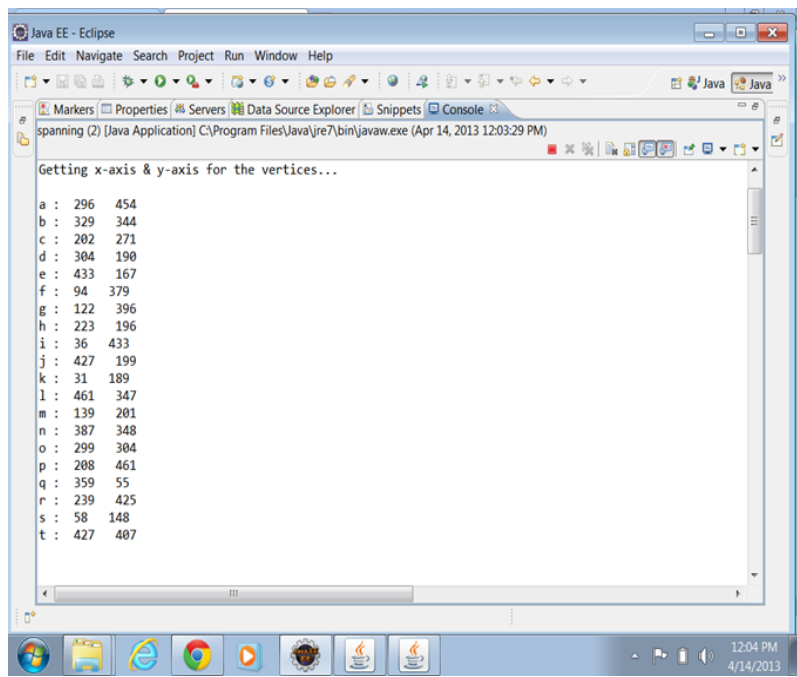

Fig 3.12: Adjacency Matrix of the Network.

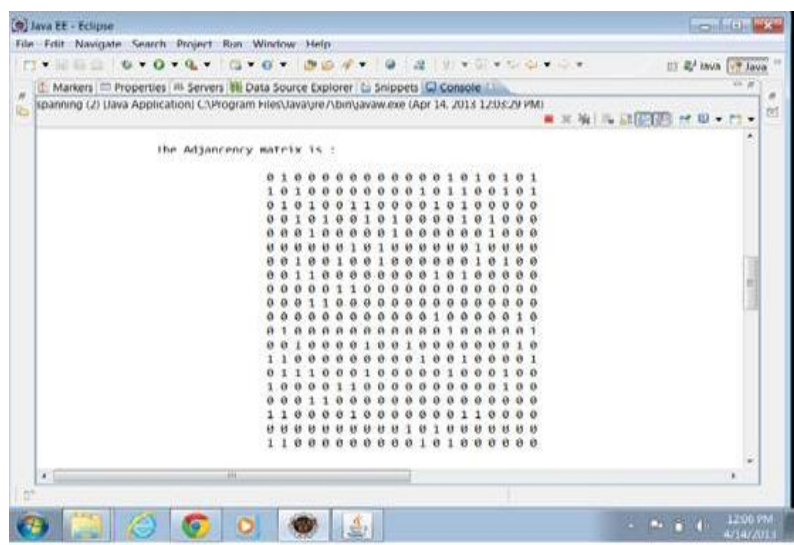

\section{Degree of the nodes}

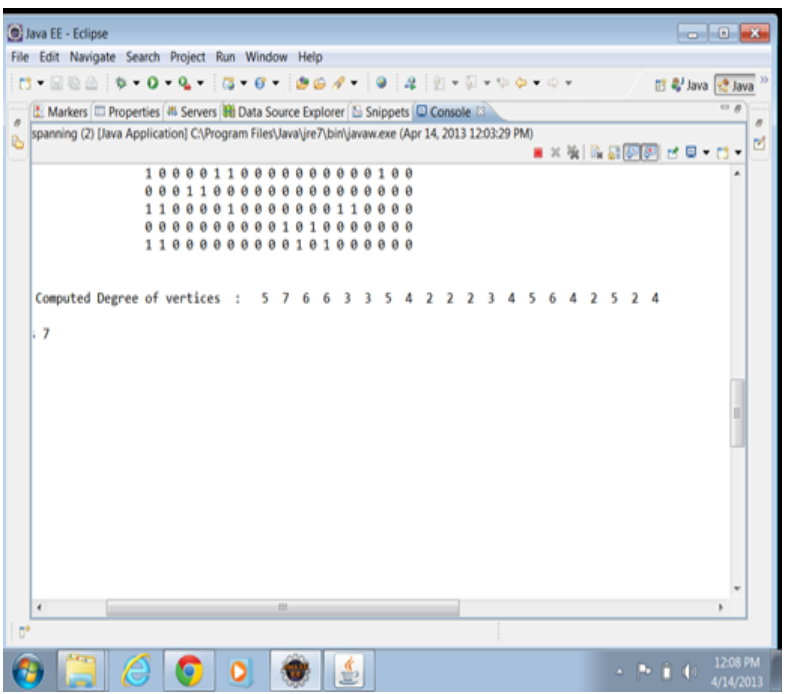

\section{Maximum degree of the nodes}
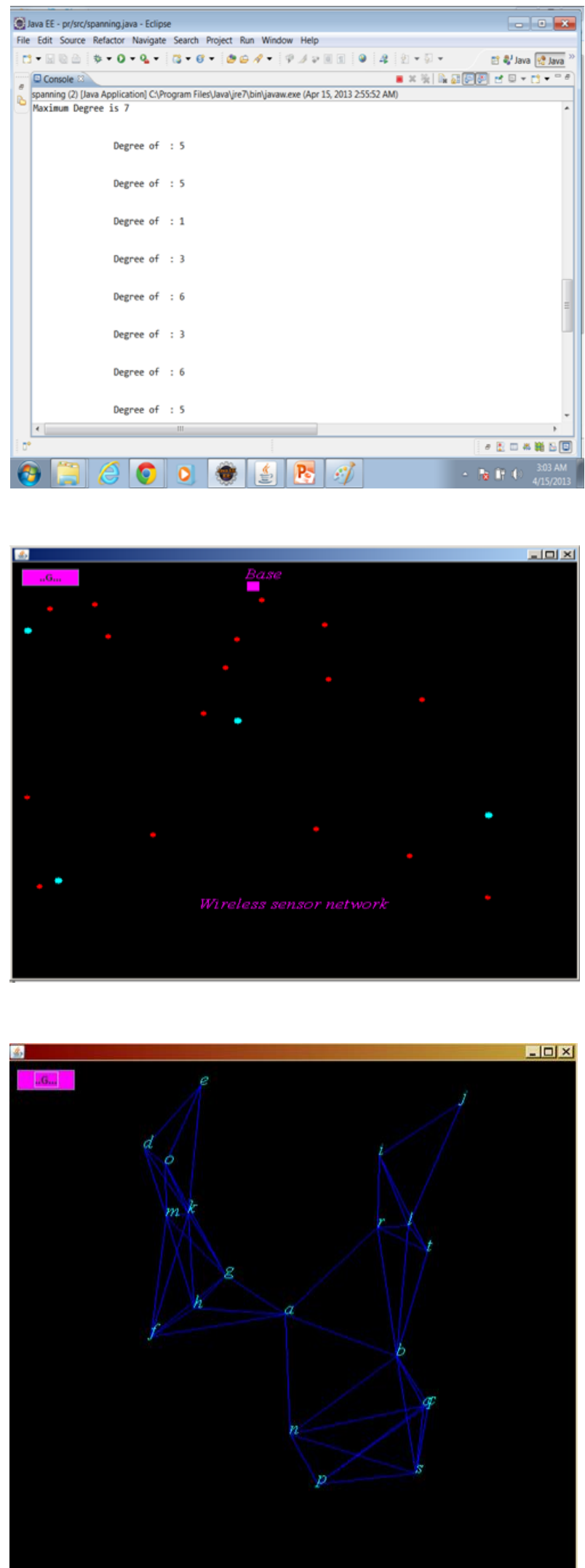
Minimum Connected Dominating Set

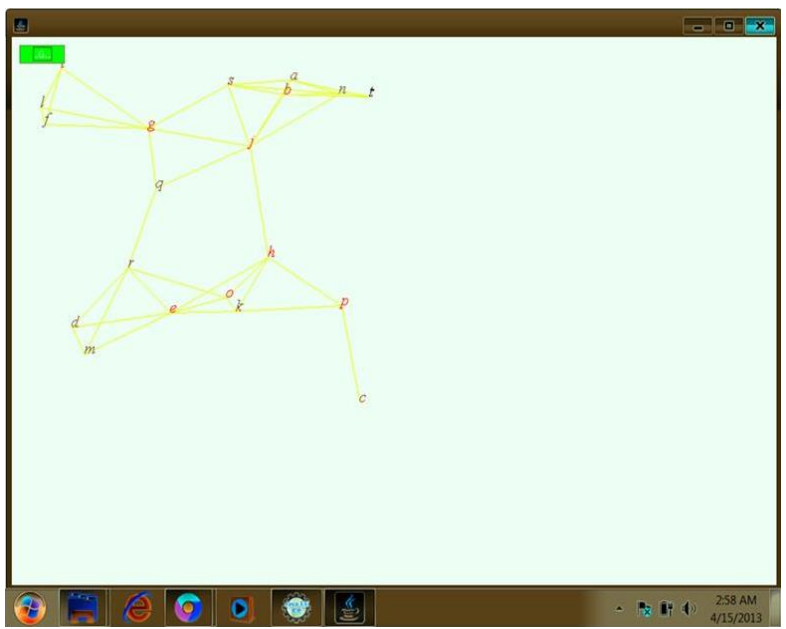

\section{CONCLUSION}

The Minimum Connected Dominating Set (MCDS) act as virtual backbone in Wireless Sensor Networks. In this project work, an algorithm for finding MCDS using the concept of convex hull is studied. Another algorithm for finding power aware MCDS is also studied and implemented.
A new algorithm for finding a routing using MCDS in wireless sensor network is proposed. Proposed algorithm is implemented and tested using Java-Eclipse. The sample outputs are also included

\section{REFERENCES}

Li, Y., S. Zhu., M. Thai and D. Du, (2004). Localized Construction of Connected DominatingmSetin Wireless Network. In Proceedings. TAWN, 1 9.

Purohit, G.N. and U. Sharma. (2010), Constructing Minimum Connected Dominating Set: Algorithmic approach, International Journal on applications of graph theory in wireless ad hoc networks and sensor networks (GRAPH-HOC) 2(3): 59-66.

Thai,T. and Ding-Zhu Du, (2006). Connected Dominating Sets in Disk Graphs with bidirectional links", IEEECommunication Letters, 10(3): 1 - 10.

Wan, P., K. Alzoubi, and O. Frieder, (2002). Distributed construction of connected dominating set in wireless adhoc networks, In Proceedings. INFOCOM. 